\title{
Aprendizagem baseada em jogos digitais: a contribuição dos jogos epistêmicos na geração de novos conhecimentos.
}

\author{
Samara de Sena \\ Programa de Pós-Graduação em Engenharia e Gestão do conhecimento, Universidade Federal de Santa \\ Catarina, senasami@gmail.com \\ Sarah Schmithausen Schmiegelow \\ Programa de Pós-Graduação em Engenharia e Gestão do conhecimento, Universidade Federal de Santa \\ Catarina, sarahschmiegelow@gmail.com \\ Gladys M. B. C. do Prado \\ Programa de Pós-Graduação em Engenharia e Gestão do conhecimento, Universidade Federal de Santa \\ Catarina,gladysmprado@gmail.com \\ Richard Perassi Luiz de Sousa \\ Programa de Pós-Graduação em Engenharia e Gestão do conhecimento, Universidade Federal de Santa \\ Catarina, richard.perassi@uol.com.br \\ Francisco Antonio Pereira Fialho \\ Programa de Pós-Graduação em Engenharia e Gestão do conhecimento, Universidade Federal de Santa \\ Catarina, fapfialho@gmail.com
}

Resumo: Para que a aprendizagem seja efetiva e atraente para as gerações atuais, são necessários recursos interativos e engajadores semelhantes aos que os estudantes estão familiarizados em suas rotinas, fazendo com estes se interessem pelos conteúdos propostos. Nesse contexto, este estudo de natureza exploratória buscou, através de pesquisa bibliográfica, conhecer os estudos acadêmicos que tratam da aprendizagem baseada em jogos digitais, enfocando, principalmente, nos estudos relacionados ao desenvolvimento e implementação de jogos epistêmicos. Concluiu-se que a aprendizagem baseada em jogos digitais surge como uma tendência que está em plena consolidação em diversas partes do mundo e que os jogos epistêmicos, foco principal deste estudo, são jogos que utilizam epistemologias próprias das mais diversas áreas do conhecimento, dentro de jogos de simulação de práticas profissionais. Estes jogos visam estimular os estudantes a pensar de acordo com esses quadros epistêmicos, da mesma forma que os profissionais pensam dentro de suas profissões.

Palavras-chave: aprendizagem baseada em jogos digitais, jogos epistêmicos, cognição situada, educação.

\section{Digital game-based learning: epistemic games contribution}

Abstract: To promote an effective and attractive learning for the current generation the use of interactive and engaging resources, as the students are familiar in their routines, is necessary to turning the proposed contents interesting for them. In this context, this exploratory study aimed, through bibliographical research, meet academic studies about the digital game-based learning, focusing mainly on studies related to the development and implementation of epistemic games. It was concluded that the digital game-based learning emerges as a trend in full consolidation around the world and that the epistemic games, this study main focus, are games that use particular epistemologies from various fields of knowledge within simulation games of professional practices. These games aim to encourage students to think according to these epistemic frames, as professionals think within their practice.

V. $14 \mathrm{~N}^{\mathrm{o}} 1$, julho, 2016 
Keywords: digital game-based learning, epistemic games, situated cognition, education.

\section{INTRODUÇÃO}

Tecnologias digitais estão cada vez mais incorporadas nas rotinas de todos. Entre estas tecnologias, os jogos digitais despontam como uma tendência e são produzidos em diversos estilos e níveis de dificuldade, abrangendo públicos das mais variadas idades. Eles deixam de ser um entretenimento exclusivo de uma minoria, que domina os jogos hardcore, para tornarem-se disponíveis a todos. Acompanhando esta tendência, a aprendizagem baseada em jogos digitais se expande, diversifica e adapta a diversos contextos de ensino-aprendizagem.

As abordagens para o uso de jogos digitais na aprendizagem são numerosas e abrangem desde a produção de jogos lúdicos, feitos exclusivamente para o ensinoaprendizagem de conteúdos escolares, até a apropriação de elementos de game design em contextos que não usam necessariamente jogos digitais, como ambientes analógicos ou virtuais de ensino e aprendizagem. Os jogos trazem em si uma infinidade de conceitos que estimulam a aprendizagem, pois permitem a interação com o meio e a construção coletiva de conceitos e experiências, bem como a geração de conhecimento. A ideia de aprendizagem por meio da interação foi muito discutida por Vigotski (2007).

Para que os jogos sejam ferramentas efetivas de ensino e aprendizagem é necessário que o sistema de educação se adapte às necessidades de um mundo que anseia por uma educação que estimule a formação do pensamento criativo e inovador, que os jogos tem potencial para ajudar a promover. As escolas atuais ainda reproduzem padrões que foram criados para atender necessidades sociais e econômicas de um certo contexto, porém, os tempos mudaram e a escola precisa ser reformulada para atender as demandas atuais. Nesse sentido, a educação deve ser desvinculada do pensamento padronizador, que ainda é o eixo norteador tradicional das escolas (Shaffer, 2006-a).

Uma vertente que se destaca entre os jogos feitos para a aprendizagem são os jogos epistêmicos. Estes jogos "são ambientes de aprendizagem digitalmente suportados, projetados de forma a permitir que os estudantes desenvolvam competências de domínio específico sob restrições realistas" (Rupp; Mislevy; Shaffer, 2010, p. 5). Ao jogar estes jogos, os estudantes são apresentados a epistemologias próprias de determinadas profissões, denominadas por Shaffer (2006-a) como "quadros epistêmicos". Estes são formadas por "coleções de habilidades, conhecimentos, identidades, valores, e epistemologias, os quais os profissionais usam para pensar de forma inovadora" (Shaffer, 2006-a, p.12). Sendo assim, jogos epistêmicos são projetados para que os jogadores aprendam a pensar inovadora e criativamente, coerente com o perfil profissional, demandado por um mundo que valoriza não mais a padronização e repetição, mas sim a inovação e o conhecimento.

Nesse contexto, esta pesquisa visa conhecer a produção acadêmica que vem sendo empreendida acerca dos jogos epistêmicos, buscando esclarecer os conceitos relacionados ao tema, bem como suas aplicações dentro da aprendizagem baseada em jogos digitais.

\section{Metodologia}

Este trabalho caracteriza-se quanto aos seus objetivos como um estudo exploratório, para tanto, utiliza métodos qualitativos de pesquisa. Segundo Gil (2008), as pesquisas exploratórias procuram obter uma visão geral, de modo aproximado, de determinado fato. Como procedimento metodológico principal utilizou-se a revisão bibliográfica de literatura, visando identificar conceitos norteadores e trabalhos 
científicos recentes que tratam do tema da aprendizagem por meio dos jogos epistêmicos para, desta forma, fundamentar as reflexões desta pesquisa em autores já consagrados e que têm autoridade sobre o tema (Motta-Roth; Hendges, 2010).

Para a fundamentação da pesquisa foram consultados livros publicados por autores que trabalham com as temáticas de aprendizagem, jogos digitais, aprendizagem baseada em jogos digitais e jogos epistêmicos, buscando identificar os conceitos principais, que nortearam o restante da pesquisa. Em um segundo momento, como o conceito de jogos epistêmicos que permeia esta pesquisa provêm dos estudos empreendidos pelo autor David W. Shaffer e demais pesquisadores do grupo Epistemic Games (1), foi realizada uma pesquisa no site do grupo, buscando mapear os trabalhos publicados pelos mesmos a respeito do tema.

Por fim, através de bases e portais como SCOPUS, ERIC e CAPES foram pesquisados estudos recentes que tratam dos jogos epistêmicos e sua relação com a aprendizagem. Sendo assim, este estudo aborda a aprendizagem baseada em jogos digitais, focando na aprendizagem através dos jogos epistêmicos.

\section{APRENDIZAGEM, EDUCAÇÃO E CONHECIMENTO}

A aprendizagem na perspectiva construtivista e social-interacionista acontece na relação com o mundo: consigo, com as pessoas e com as experiências. A aprendizagem social dá valor a relação entre o indivíduo e o contexto social. E, a construtivista, percebe que a aprendizagem surge da construção do sujeito a partir da sua interação com o meio. O significado é constituído por quem aprende e depende da sua estrutura de conhecimentos prévios (Pedrosa, 2014). Fialho (2011-a) afirma que a aprendizagem que ocorre no indivíduo é produto de uma construção sócio-interativa intra e interpessoal. Só assim é possível para o indivíduo construir seus significados e seu conhecimento.

Segundo Piaget (2004) a aprendizagem só ocorre quando a criança já tem sua maturação física/psíquica para o tema em questão. Por meio da epistemologia genética ele descreveu como as crianças atravessam diferentes estágios de desenvolvimento e como elas pensam durante esses estágios, até que estejam prontas para entender o mundo em termos abstratos (Shaffer, 2006-a). Em cada uma dessas quatro fases a criança aprende a lidar com diferentes situações e se prepara para o próximo estágio. Não adianta desafiá-la a solucionar problemas para os quais ainda não está madura. As idades podem sofrer variações, mas nunca uma fase se antecipará à outra. Na teoria de Piaget (2004) o meio exterior pode e influencia, porém somente após o nível de desenvolvimento interno ter atingido um estágio mínimo de desenvolvimento e ter uma base para que o novo conhecimento seja compreendido. Fialho (2011-b) lembra que Piaget também dizia não haver cognição sem emoção, e nem emoção sem cognição, o cognitivo indica para o indivíduo aonde ir, enquanto que só a emoção dá a energia que permite o movimento. Ou seja, é necessário motivação para aprender e se engajar no aprendizado.

Por sua vez, Vigotsky (2007) afirma que as experiências formativas são culturais e os indivíduos constroem sua identidade e cognição pela relação com os outros. Ele contribuiu para a mudança do enfoque de aprendizagem focada na criança para a aprendizagem colaborativa (Shaffer, 2006-a). Nesta perspectiva o indivíduo se constitui e se desenvolve na medida em que interage e é estimulado pelo meio.

Vigotsky (2007) acredita na inter-relação do aprendizado e do desenvolvimento desde o primeiro dia de vida do indivíduo, embora frise que não são a mesma coisa e nem ocorrem em paralelo. Para ele a aprendizagem antecede o desenvolvimento. Um dado postulado empiricamente e já bem disseminado para o autor é que o aprendizado 
carece de ser compatível com o nível de desenvolvimento da criança. A partir destes dados, Vigotsky desenvolveu o conceito de zona de desenvolvimento proximal (ZPD) que é a extensão entre o nível de desenvolvimento real, determinado comumente por meio da solução de problemas de forma independente, isto é, sem colaboração de outra pessoa ou material, e o nível de desenvolvimento potencial, verificado por meio da solução de problemas com a orientação ou colaboração de outras pessoas, sejam adultos ou colegas de escola mais capazes para a tarefa em questão (Vigotsky, 2007).

Tem-se aqui um embasamento para atividades de aprendizagem que ocorrem por meio da imitação, orientação, mentoria, supervisão e mesmo o trabalho em grupo ou coletivo, acelerando um processo de desenvolvimento que demoraria mais tempo para ocorrer, caso não fosse estimulado pelo meio.

Nash e Shaffer (2011) realizaram um estudo sobre o impacto da mentoria de profissionais mais experientes no aprendizado de jovens por meio de um jogo epistêmico, o Urban Science. A pesquisa apresenta resultados diretos, embora tímidos, sobre a aprendizagem por imitação, uma vez que além da imitação imediata, ele gera a internalização do conceito imitado e da tarefa em si. Baseados nos conceitos de Vigotsky, definem como internalização a capacidade de manter o aprendizado e aplicálo em situações futuras, mesmo após ter passado um tempo do experimento e sem a presença do mentor.

Pedrosa (2014) e Shaffer (2006-a), ao falarem sobre aprendizagem, abordam Dewey, que desenvolveu o conceito de aprendizagem através da experiência. Seu enfoque está no poder das atividades autênticas, ou seja, sobre a aprendizagem que acontece por meio das atividades experienciadas e praticadas. Shaffer (2006-a) destaca que desde o século passado, já se afirmava, com base no movimento progressivo de Dewey, que as crianças deveriam aprender por meio da solução de problemas significativos, baseados em seus próprios trabalhos.

Dewey (1971 apud Pedrosa, 2014) apresentou o princípio da continuidade por meio do fluxo: situação > problema > indagação > reflexão > nova situação. Isto é, todas as experiências vividas por um indivíduo estão interligadas entre si, pois toda experiência toma algo das experiências anteriores e modifica de alguma forma as experiências seguintes (Pedrosa, 2014). Dito isto, fica evidente a importância de considerar o nível de desenvolvimento do indivíduo e utilizar diferentes formas para propiciar sua aprendizagem, o que impacta diretamente no modelo de educação que gera maior resultado.

Shaffer (2006-a) provoca a reflexão sobre a crise na educação e chama a atenção para o fato de que as escolas no formato que conhecemos hoje foram idealizadas nos EUA para educar o grande número de migrantes dos campos para as cidades, no período da Revolução Industrial. O modelo que se tem ainda hoje nas escolas foi desenvolvido no final do século XIX e prepara operários para seguirem instruções, ordens e serem submissos. Isto é, a educação formal ainda hoje forma as pessoas para a ordem, um comportamento adaptado a um padrão geral de respeito horários e hierarquia. Ter sua tarefa pronta na data, cumprir o cronograma predeterminado e atingir as metas de produção. Um modelo que não atende mais as necessidades do mundo atual.

É necessário nos dias atuais, qualificar pessoas para a educação contínua, e não mais para situações padronizadas, pois precisarão ser capacitadas em tecnologias que logo estarão obsoletas. Fialho (2011-b) afirma que é necessário desenvolver pessoas para criar, transformar e organizar diferentes tipos de conhecimentos, estimulando-as a utilizarem de forma sábia as informações e conhecimentos disponíveis.

Shaffer (2006-a) aponta que o grande problema deste modelo educacional é não encorajar o pensamento inovador, a criatividade. Em uma era de competição global, não 
há mais a necessidade de conhecimentos e habilidades padronizados. Ainda não está claro o que os trabalhadores precisarão fazer no futuro, mas sabe-se que a maior parte dos trabalhos terão que ser basicamente intensivos em conhecimento (Davenport apud Shaffer, 2006-a).

$\mathrm{Na}$ direção de trabalhar para esta necessidade de educação contínua da sociedade que se vive, os conceitos de aprendizagem situada e comunidade de prática tem sido muito explorados. Segundo Fialho (2011-a) a aprendizagem é um fenômeno situado que resulta de um processo progressivo de participações em uma comunidade de prática, ou seja, é a construção sócio-interativa intra e interpessoal. Isto é, a aplicação dos conceitos discutidos por Vigotsky na vida adulta, não somente no desenvolvimento infantil.

A aprendizagem situada envolve algumas características: a observação de pessoas mais habilidosas; o aculturamento pela linguagem em tarefas; a explicitação do tácito; a introjeção dos termos e significados com seus respectivos valores associados às ações de quem está realizando; e o aprender fazendo (Fialho 2011-a). O importante é que o conhecimento seja apresentado e aprendido em um contexto real, com os elementos e aplicações que naturalmente envolveriam esse conhecimento. A aprendizagem requer interação social e colaboração (Lave, 2003 apud Fialho, 2011-a). A motivação em aprender algo com aplicação real é maior, uma vez que está diretamente relacionado à emoção do aprendiz e a sua necessidade presente.

As comunidades de prática permitem este exercício de aprendizado, uma vez que se baseiam em uma atividade na qual um grupo de pessoas com interesses $\mathrm{e}$ conhecimentos comuns em certo tema compartilham práticas mutuamente negociadas (Fialho, 2011-a). Aprendizagem é uma questão ligada ao fato de pertencer e participar. A partir desses conceitos é possível discutir a relevância dos jogos digitais para os desafios que a sociedade enfrenta em relação à educação.

\section{APRENDIZAGEM BASEADA EM JOGOS DIGITAIS}

A aprendizagem baseada em jogos digitais é uma tendência que vem sendo incorporada cada vez mais na educação. Johnson et al. (2012, 2014a, 2014b, 2014c) apontam para a entrada imediata de games, gamificação e aprendizagem baseada em jogos digitais nas escolas e universidades do Brasil, nas escolas internacionais da Ásia e nas escolas da Europa.

A apropriação dos jogos e seus elementos em contextos educacionais pode ser vista em diversas configurações. Em jogos projetados especificamente para a educação, conhecidos como jogos educacionais, jogos educativos ou edutenimento, conteúdo e jogabilidade são criados especificamente para abordar temas escolares. Já a gamificação utiliza elementos e dinâmicas dos jogos, como níveis, progressões e pontuações, de forma lúdica, em ambientes analógicos ou virtuais de aprendizagem (Alves, 2014, p.

26). Em outra abordagem, editores de jogos são utilizados para a criação autoral e muitas vezes colaborativa por parte de alunos e professores.

A aprendizagem baseada em jogos digitais é eficaz por que utiliza técnicas de aprendizagem interativa, que não provém de jogos, associadas a técnicas de aprendizagem interativa que já vêm sendo implementadas em jogos comerciais desde os primeiros jogos desenvolvidos. Entre essas técnicas destacam-se: prática e feedback, aprender na prática, aprender com os erros, aprendizagem guiada por metas, aprendizagem guiada pela descoberta, aprendizagem baseada em tarefas, aprendizagem guiada por perguntas, aprendizagem contextualizada, role-playing, treinamento, aprendizagem construtivista, aprendizagem acelerada, selecionar a partir de objetos de aprendizagem e instrução inteligente (Prensky, 2012). Além disso, a aprendizagem baseada em jogos digitais é eficiente porque está de acordo com o estilo de 
aprendizagem dos estudantes atuais e futuros, é motivadora, por ser divertida e é bastante versátil porque pode ser adaptada a quase todas as disciplinas e habilidades a serem aprendidas, sendo muito eficaz se for corretamente utilizada (Prensky, 2012).

No entanto, apesar das inúmeras possibilidades disponíveis dentro da aprendizagem baseada em jogos digitais, nem todo aplicativo de aprendizagem digital é necessariamente um jogo. Para que um jogo possa ser considerado um "jogo", algumas características devem ser preservadas. Misfeldt (2015) aponta que os jogos sempre comunicam o fato de serem jogos, por implicar simulações, representações de ações ao invés de ações, e envolver a competição para avançar em relação a outros jogadores ou aumentar a pontuação em uma escala artificial. Macgonigal (2011) defende que, para que algo possa ser considerado um jogo, é importante que contenha quatro elementos essenciais: objetivos, regras, sistemas de feedback e participação voluntária. Os objetivos são os resultados específicos que os jogadores precisam atingir. As regras criam as limitações de como os jogadores podem atingir os objetivos. Os sistemas de feedback informam os jogadores quão perto eles estão de atingir os objetivos, podendo estes aparecer em forma de pontuações, níveis, placares ou barras de progresso. Já a participação voluntária se refere ao fato de que todos os jogadores aceitam propositalmente e de bom grado os objetivos, as regras e os feedbacks do jogo que estão participando (Macgonigal, 2011).

Para Shaffer (2006-a), o que o que faz de um jogo um "jogo" não é nem a diversão, nem as condições de vitória ou derrota, mas sim o fato de que um jogo possui um certo conjunto de regras que o jogador precisa seguir. Outro conceito é lançado por Abt (1970, p.9, apud Boots \& Strobel, 2014) que define jogo como "uma atividade entre dois ou mais decisores independentes que buscam atingir seus objetivos em um contexto limitador. Uma definição mais convencional diria que um jogo é um contexto com regras entre adversários tentando alcançar objetivos".

Além de contemplarem aspectos inerentes aos demais tipos de jogos digitais, jogos feitos para a aprendizagem precisam atentar para fatores como a manutenção do interesse e da motivação dos estudantes e, ao mesmo tempo, precisam garantir o correto entrelaçamento entre os objetivos instrucionais e a jogabilidade. A simples inserção de conteúdo educacional em jogos de entretenimento não é garantia de que o jogo será bem sucedido nos seus objetivos educacionais (Boots; Strobel, 2014). Nesse contexto, Gunter et al. (2008 apud Boots; Strobel, 2014) apontam que o impacto de um jogo educacional deve ser analisado em termos do quanto ele insere o conteúdo acadêmico dentro do contexto da fantasia do jogo e de como os designers do jogo combinam o jogo com outras estratégias instrucionais. Os jogos educacionais capazes de impactar a visão de mundo de uma criança são os que a engajam com um aprendizado complexo e profundo desde o início (Nodoushan, 2009 apud Boots; Strobel, 2014). Cenários nos quais artefatos de baixa qualidade são disponibilizados para os estudantes, mas que não são atraentes para um público acostumado com atividades mediadas pela tecnologia, podem não despertar o correto engajamento e o prazer de interagir com esses jogos (Alves, 2008). Além disso, Prensky (2012) atenta para o fato de que o estudante e o conteúdo devem ser bem compatibilizados, do contrário, a aprendizagem pode ser prejudicada.

Sendo assim, jogos possuem potencial educacional, são engajadores, permitem que os usuários façam testes em um ambiente artificial e despertam nos jogadores o esforço constante para serem melhores (Gee, 2003 apud Misfeldt, 2015), além disso, possuem a vantagem de permitir que os usuários executem ações em uma prática simulada (Shaffer, 2006 apud Misfeldt, 2015). 


\section{JOGOS EPISTÊMICOS E APRENDIZAGEM}

Os jogos epistêmicos são indicados como uma oportunidade para os educadores avançarem nos métodos educacionais, que foram constituídos ainda durante a revolução industrial. Por meio desta modalidade de jogos, os estudantes aprendem a partir da participação em simulações autênticas de práticas profissionais (Shaffer, 2006-b).

Bagley e Shaffer (2009, p.36 apud Boots; Strobel, 2014) definem jogos epistêmicos como "jogos onde os jogadores pensam e agem como profissionais do mundo real", aplicando informações em um contexto significativo, em um ambiente similar a um jogo. Para os autores, estes são em essência jogos de interpretação de papéis, que permitem aos jovens aprender conhecimentos, habilidades e valores de uma profissão através de um treinamento profissional simulado.

Os jogos epistêmicos procuram equipar os jogadores com a habilidade de pensar de modo inventivo (Boots; Strobel, 2014). Nas simulações dos jogos epistêmicos, os aprendizes interpretam o papel de estagiários em empresas, interagem com chefes virtuais, e podem resolver problemas do mundo real sem a necessidade de dominar conteúdos básicos do domínio, pois o conhecimento e as habilidades que os estudantes ainda não possuem estão incorporadas nas ferramentas usadas no jogo (Arastoopour; Chesler; Shaffer, 2014).

Rupp, Mislevy e Shaffer (2010) apontam que os jogos epistêmicos baseiam-se em teorias de aprendizagem da era digital. Neste sentido, explicam que estes jogos são ambientes de aprendizagem digitalmente suportados, projetados para que os estudantes possam desenvolver competências em um domínio específico, sob restrições realistas.

Através de um jogo epistêmico, estudantes aprendem a pensar e agir como jornalistas, artistas, gerentes de negócios ou engenheiros. Para tanto, é projetado um jogo que imita as principais experiências pelas quais os estudantes passariam se estivessem em um estágio profissional no campo. Desta forma, estes jogos permitem experiências caracterizadas por uma mescla de trabalho individual e colaborativo, tanto na vida real como no ambiente virtual (Rupp et al., 2010).

A práxis pedagógica estende o conceito de comunidades de prática de Lave e Wenger ao sugerir que cada comunidade de prática possui um quadro epistêmico distinto. Shaffer define quadros epistêmicos como diferentes modos de saber, decidir o que vale a pena saber, e acrescentar ao conhecimento e entendimento coletivo da comunidade. O quadro epistêmico de uma profissão é a combinação de valores, conhecimento, habilidades, epistemologia e identidade usada pelos profissionais para resolver problemas no contexto de atuação profissional (Shaffer, 2006-a).

Os quadros epistêmicos são exemplificados por Shaffer (2006-b) explicando que geômetras, economistas, estatísticos e engenheiros são profissionais que usam a matemática. No entanto, cada um possui um quadro epistêmico distinto, que incorpora entendimentos e estruturas epistêmicas diferentes do domínio da matemática. Portanto, o autor conclui que o desenvolvimento de expertise implica no desenvolver expertise de algum modo particular, a partir de alguma perspectiva particular, e relacionado ao modo saber de certa comunidade de prática.

Uma vez que os estudantes aprendem a pensar como determinados profissionais, eles são capazes de usar as formas de saber aprendidas em outros contextos. Desta forma, o autor argumenta que as experiências em simulações de práticas profissionais do mundo real assistidas por computador, que permitem a incorporação de quadros epistêmicos de determinada comunidade de prática, podem ajudar os estudantes a lidarem com situações do mundo real de modo mais efetivo, e também melhorar a capacidade dos estudantes em outras disciplinas além do escopo do ambiente interativo experienciado (Shaffer, 2006-b). 
Sensivy (2012) aborda o conceito de jogos de aprendizagem como uma maneira de modelar o que o professor e o aluno fazem em conjunto para que o aluno aprenda algo. $\mathrm{O}$ autor conecta esse conceito aos jogos epistêmicos afirmando que um jogo epistêmico é uma maneira de modelar o conhecimento que tem que ser aprendido pelo aluno. "Um jogo epistêmico é a modelagem do que podemos chamar de um conhecimento prático, uma prática baseada em habilidades específicas que fazem essa prática eficiente" (Sensivy, 2012, p.512).

Jogos epistêmicos diferem de outros jogos em vários aspectos. Rupp et al (2010) atentam para o fato de que, diferente do que acontece em um jogo de entretenimento, nos jogos epistêmicos o núcleo do jogo é baseado em profissões da vida real e apenas aspectos relacionados a essas profissões são desenvolvidos. Já em jogos de entretenimento, mesmo em jogos de simulação como o The Sims, vários aspectos são abordados de forma mais geral. Os autores apontam ainda que os jogos epistêmicos não são projetados para orientação profissional e treinamento, mas para prover situações que levem os estudantes a pensar e agir de acordo com determinada área do conhecimento. Outro aspecto que diferencia os jogos epistêmicos dos demais jogos é o fato de que as decisões são tomadas dentro de restrições reais da rotina da profissão e em tempo real, enquanto em jogos de entretenimento aspectos como tempo, recursos e decisões podem ser manipulados (Rupp et al., 2010).

Shön (1983, 1987 apud Nash; Shaffer, 2011) aponta que um elemento crítico para a prática profissional é a interação que se dá entre os jovens aprendizes e seu mentores, considerada positiva para influenciar o desenvolvimento dos jovens, destacam Nash e Shaffer (2011). Neste contexto, os autores indicam a interação entre mentores e estudantes nos jogos epistêmicos como uma possibilidade de reprodução da modelagem da forma de pensar de uma profissão que ocorre nas práticas profissionais.

Como exemplo deste tipo de interação em jogos epistêmicos, os autores apresentam o já citado jogo Urban Science, onde os estudantes interpretam o papel de planejadores urbanos encarregados de redesenhar bairros de sua cidade. Neste jogo, os estudantes interagem regularmente com os chamados "consultores de planejamento", profissionais experientes que conversam, dão suporte, feedback e inspiração aos estudantes nas tarefas do jogo. Os resultados do estudo mostraram que a interação com os mentores contribuiu no desenvolvimento dos quadros epistêmicos dos estudantes. Apesar dos resultados serem específicos do estudo feito com o jogo epistêmico Urban Science, os autores indicam que o modo de aprendizagem e mentoria observado nestes jogos pode servir como modelo para o desenvolvimento de novos contextos de aprendizagem.

Uma das aplicações mais efetivas de jogos epistêmicos acontece na área de engenharia, pois, através desses jogos, é possível proporcionar aos estudantes a vivência prática da profissão, sem que grandes quantidades de recursos financeiros, físicos e de pessoal sejam despendidos. Nesse sentido, Chesler et al. (2013), relatam a experiência de estudantes de engenharia com o jogo Nephro Tex. Nele, times de estudantes dos primeiro ano de engenharia, de uma Universidade pública americana, foram guiados através de ciclos de tarefas que envolviam a concepção e a construção de estruturas, dentro de um estágio virtual. Este estudo demonstrou como a vivência profissional dentro de um estágio virtual é motivadora para os estudantes, pois garante que estes tenham acesso a situações práticas já desde o primeiro ano de curso. Outro estudo, de Arastoopour et al. (2015), demonstra que o aprendizado de engenharia através de jogos epistêmicos também melhora o desenvolvimento do pensamento complexo dos estudantes. Em seu estudo, os autores utilizaram o método ENA (epistemic network analysis) para a medição sobre a forma como o pensamento complexo é desenvolvido através do tempo, dentro de um estágio virtual de engenharia. Vivenciar os desafios 
profissionais de engenharia dentro de estágios virtuais em jogos epistêmicos oferece a oportunidade para os estudantes de se envolver em projetos de engenharia autênticos, receber feedbacks simultâneos no seu design, e desenvolver a identidade, valores e formas de pensar de engenheiros do século 21 (Arastoopour et al. 2015).

\section{CONSIDERAÇÕES FINAIS}

A sociedade do século XXI necessita de pessoas criativas, inovadoras e produtivas, e para desenvolvê-las é imprescindível que os processos de aprendizagem oferecidos pelos sistemas educacionais sejam revistos. Nesta pesquisa foi possível compreender pontos relevantes sobre como ocorre a aprendizagem e a geração de novos conhecimentos.

Para que a aprendizagem seja efetiva, deve ser adequada às demandas de seu tempo e de seu público. Desde Piaget e Vigostsky sabe-se da necessidade de adequar o conteúdo e forma ao momento de desenvolvimento das pessoas. Além disso, outros autores reforçam que ao abordar temas relacionados com a realidade, focados na necessidade presente e na motivação do indivíduo tem-se resultados superiores na retenção e eficácia do aprendizado.

Dessa forma, para as novas gerações de estudantes já acostumadas a utilizar jogos todos os dias, a aprendizagem baseada em jogos digitais é uma forma de aprendizagem mais coerente com a sua realidade. "Comparada com jogos, a realidade é muito fácil. Jogos nos desafiam com obstáculos voluntários e nos ajudam a aplicar nossas forças pessoais para uma melhor utilização" (Macgonigal, 2011, p. 22).

Pode-se dizer também que, de modo direto, o jogo cria um ambiente de aprendizagem que incorpora conteúdo acadêmico de modo fluido e que engaja os estudantes, proporcionando oportunidades para a construção do conhecimento para um futuro no qual o aprendizado será ainda mais técnico e complexo.

Os jogos epistêmicos são os mais notáveis entre uma série de novos videogames sérios ou educacionais, que visam ensinar para o jogador habilidades necessárias em profissões que requerem processos complexos de pensamento. Isto se dá pelo estímulo do modo de pensar em um contexto particular. Afinal, aprender uma disciplina específica não se restringe ao aprendizado dos conceitos e procedimentos. É necessária também a capacidade de pensar, agir e interagir com outros de modo produtivo, bem como a capacidade de resolução de tarefas complexas em situações similares as do mundo real.

Porém, apesar da potencialidade dos jogos epistêmicos para a aprendizagem, os estudos indicam que a criação destes jogos poderá ser limitada a campos convencionais como medicina, direito, ciência e educação, pois, pelas dificuldades de se criar simulações para determinados ambientes profissionais, há indícios de que algumas áreas não serão contempladas com estes jogos.

Tendo em vista que a aprendizagem baseada em jogos digitais já está se estabelecendo nas escolas de todo o mundo, a necessidade de se criar jogos de qualidade, que contemplem o aprendizado de campos específicos do conhecimento e, além disso, incentivem a criatividade e o pensamento inovador, é uma demanda urgente que se faz cada vez mais necessária nos processos de ensino e aprendizagem contemporâneos.

Nota: (1) Disponível em: http://edgaps.org/gaps/. Acesso em 03 set. 2015.

\section{Referências}


ALVES, Flora. Gamification: como criar experiências de aprendizagem engajadoras um guia completo: do conceito à prática. São Paulo: DVS editora, 2014.

ALVES, L. Relações entre os jogos digitais e aprendizagem: delineando percurso. Educação, Formação \& Tecnologias, v.1, n.2, 2008. pp.3-10. Disponível em: < http://eft.educom.pt>. Acesso em: 24 set 2015.

ARASTOOPOUR, G., SHAFFER, D.W., SWIECKI, Z., RUIS, A. R., CHESLER, N. C. Teaching and Assessing Engineering Design Thinking with Virtual Internships and Epistemic Network Analysis. Harvey Mudd Design Workshop. Claremont, CA, 2015.

ARAUJO, U. F. Escola, democracia e a construção de personalidades morais.

Educação Pesquisa, v.26 n.2, 2009, pp. 91-107. Disponível em: < http://dx.doi.org/10.1590/S1517-97022000000200007>. Acesso em: 29 jun 2015.

BOOTS, N.K; STROBEL, J. Equipping the designers of the future: best practices of epistemic video game design. Games and Culture, v.9, n.3, 2014. pp-167-181.

CHESLER, N., ARASTOOPOUR, G., D`ANGElO, C., BAGLEY, E., SHAFFER, D.W. Design of a professional practice simulator for educating and motivating first-year engineering students. Advances in Engineering Education, v. 3, n.3, 2013. pp.1-29.

FIALHO, F. A. P. Psicologia das atividades mentais. Florianópolis, Brasil: Editora Insular, 2011-a.

FIALHO, F. A. P. Uma escola para os magos do amanhã: um ser interdisciplinar aberto ao diálogo. Pinhais : Editora Melo, 2011-b.

GIL, Antonio Carlos. Métodos e técnicas de pesquisa social. São Paulo: Atlas, 2008.

JOHNSON, L. et al. Technology Outlook for Brazilian Primary and Secondary

Education 2012-2017: An NMC Horizon Project Regional Analysis. Austin, Texas: The New Media Consortium, 2012. Disponível em: <http://www.nmc.org/pdf/2012technology-outlook-brazilian-primary-and-secondary-education.pdf $>$. Acesso em: 21 maio 2015.

JOHNSON, L. et al. 2014 NMC Technology Outlook for Brazilian Universities: A Horizon Project Regional Report. Austin, Texas: New Media Consortium, 2014-a. Disponível em: <http://cdn.nmc.org/media/2014-nmc-technology-outlook-brazilianuniversities-PT.pdf.> Acesso em: 3 set 2015.

JOHNSON, L. et al. 2014 NMC Technology Outlook for International Schools in Asia: A Horizon Project Regional Report. Austin, Texas: The New Media Consortium, 2014-b. Disponível em: <http://cdn.nmc.org/media/2014-nmc-technologyoutlook-isa-EN.pdf> . Acesso em: 3 set 2015.

JOHNSON, L. et al. Horizon Report Europe: 2014 Schools Edition. Luxembourg: Publications Office of the European Union, \& Austin, Texas: The New Media Consortium, 2014-c. Disponível em: <http://cdn.nmc.org/media/2014-nmc-horizonreport-EU-EN.pdf>. Acesso em: 3 set 2015. 
MACGONIGAL, Jane. Reality is Broken: why games make us better and how they can change the world. London: Handom House, 2011.

MOTTA-ROTH, D. \& HENDGES, G. H. Produção textual na universidade. São Paulo: Parábola Editorial, 2010.

NASH, P.; SHAFFER, D. W. Mentor modeling: the internalization of modeled professional thinking in a epistemic game. Journal of Assisted Learning, v.27, n.2, 2011. pp. 173-189.

PEDROSA, V. M. Comunidades de prática e processos de aprendizagem: um Estudo com Coordenadores Técnicos de uma Escola Técnica - Senai. [Dissertação de Mestrado]. Universidade Federal de Pernambuco. Recife, 2014. Disponível em: $<$ http://repositorio.ufpe.br/bitstream/handle/123456789/14065/DISSERTA\%C3\%87\%C 3\%830\%20Vanessa\%20de\%20Mendon\%C3\%A7a\%20Pedrosa.pdf?sequence $=1 \&$ is All owed=y>. Acesso em: 28 ago 2015.

PIAGET, J. Seis estudos de psicologia. 24a . ed. Rio de Janeiro : Forense Universitária, 2004.

PRENSKY, Marc. Aprendizagem baseada em jogos digitais. São Paulo: Editora Senac São Paulo, 2012.

RUPP, A. A.; GUSHTA, M.; MISLEVY, R. J.; SHAFFER, D. W.. Evidence-Centered Design of Epistemic Games: Measurement Principles for Complex Learning Environments. Journal of Technology, Learning, and Assessment, v.8, n.4, 2010. pp. 1 - 48. Disponível em: 〈http://eric.ed.gov/?id=EJ873673 >. Acesso em: 20 ago 2015.

SHAFFER, David Williamson. How Computer Games Help Children Learn. New York; Palgrave MacMillian, 2006-a.

SHAFFER, D.W. Epistemic frames for epistemic games. Computers \& Education, 46, 2006-b. pp.223-234.

SENSEVY, Gérard. About the joint action theory in didacts. Zeitschrift fur Erziehungswissenschaft, v.15, n.3, 2012, Pages 503-516.

VIGOTSKY, L. S. A formação social da mente: o desenvolvimento dos processos psicológicos superiores. $7^{\mathrm{a}}$. ed. São Paulo: Martins Fontes, 2007. 